\section{MS8-P3 Structural and Biochemical Characterization of Sjögren syndrome/scleroderma autoantigen 1 (SSSCA1)}

Harmonie J.G. Perdreau-Dahl ${ }^{1,2}$, Hanne Guldsten ${ }^{2}$, Cinzia Progida $^{3}$, Magnus Arntzen ${ }^{4}$, Bernd Thiede ${ }^{4}$, Oddmund Bakke ${ }^{3}, \mathrm{~J}$. Preben Morth ${ }^{1,2}$

1. Institute for Experimental Medical Research (IEMR), Oslo University Hospital, Ullevål PB 4956 Nydalen, NO-0424 Oslo, Norway

2. Centre for Molecular Medicine Norway (NCMM), Nordic EMBL Partnership, University of Oslo, P.O. Box 1137 Blindern, 0318 Oslo, Norway

3. Centre for Immune Regulation, Department of Molecular Biosciences, University of Oslo, Blindernveien 31, 0371 Oslo, Norway

4. The Biotechnology Centre of Oslo, University of Oslo, P.O. Box 1137 Blindern, 0318 Oslo, Norway

email: harmonie.perdreau@ncmm.uio.no

Sjögren syndrome/scleroderma autoantigen 1 (SSSCA1) was discovered in the late 1990's as a novel auto-antigen over-expressed in Sjögren's syndrome and/or scleroderma patients [1]. Functionally, SSSCA1 is largely uncharacterized although it has been linked to mitosis and centromere association $[1 ; 2]$. In the past five years, it has been reported in a number of studies for its possible implication in: (i) The Wnt signaling pathway [3; 4], (ii) the cellular proliferation or stroma activation of several cancer cells [5] and (iii) identified as a key gene for pathway activity in colorectal adenoma-to-carcinoma progression [6]. In order to verify these hypotheses and to characterize SSSCA1, we have combined results from structural biology, cellular imaging techniques and proteomics.

Human SSSCA1 is a small protein of $21.5 \mathrm{kDa}$ composed of three domains: An N-terminal zinc finger domain, an intermediate proline-rich region and a C-terminal helical domain. A phylogenetic tree revealed that SSSCA1's domains architecture is evolutionary conserved. We have recently determined the crystal structure of the highly conserved zinc binding domain at a resolution of $2.8 \AA$ and showed that SSSCA1 forms a homodimer. This domain possesses a unique architecture and represents a new Pfam family of clan Zn-binding ribbon domain.

The primary structure of the C-terminus has been predicted as highly disordered [7]. SSSCA1 therefore belongs to the very large protein family of intrinsically disordered proteins (IDP's), whose function is coupled to the folding/binding with a specific partner. We have thus developed a tandem affinity purification (TAP) method coupled to cell imaging and mass spectrometry [8] to identify SSSCA1's binding partners in different cancer cell lines. We are now working on the confirmation and characterization of the five best hits using different approaches (cellular assays, human cells, yeast and E. coli expression, purification, crystallization, drug screening ...).

References:

[1]

Muro, et al., Clin Exp Immunol 111

$(1998)$ 372-6.

[2] Zhou, et al., J Immunol 167 (2001) 7126-33.

[3] Voronkov, et al., J Med Chem 56 (2013) 3012-23.
[4] Huang, et al., Nature 461 (2009) 614-20.

[5] Sillars-Hardebol, et al., J Pathol 226 (2012) 1-6.

[6] Sillars-Hardebol, et al., Tumour Biol 31 (2010) $89-96$.

[7] Ward, et al., Bioinformatics 20 (2004) 2138-9.

[8] Gingras, et al., Nat Rev Mol Cell Biol 8 (2007) 645-54

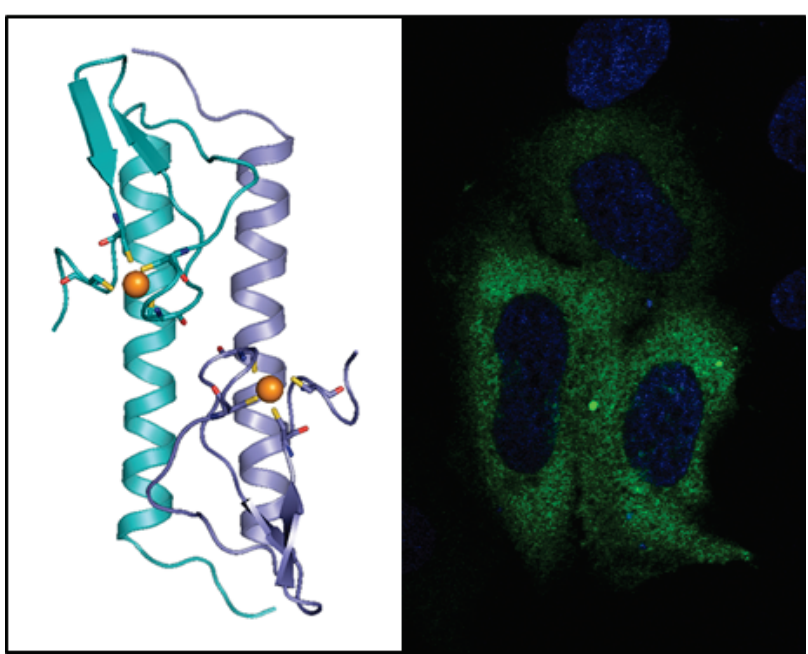

Figure 1. Crystal structure of SSSCA1's Zn-binding domain and subcellular localization in human cells.

Keywords: Crystallography, zinc binding, mitosis 